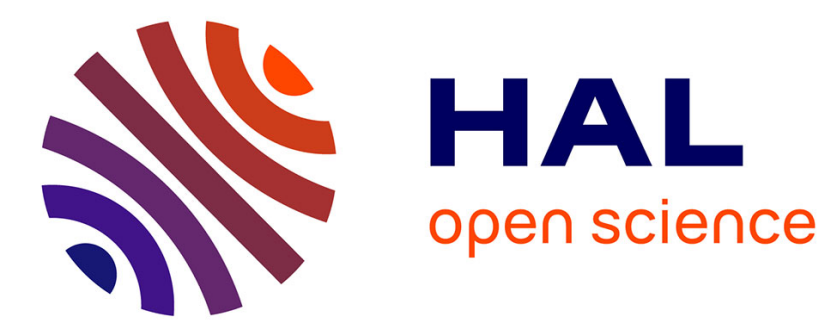

\title{
Best decay rate, observability and open-loop admissibility costs: discussions and numerical study
}

Kais Ammari, Arnaud Munch

\section{To cite this version:}

Kais Ammari, Arnaud Munch. Best decay rate, observability and open-loop admissibility costs: discussions and numerical study. 2014. hal-01071013

\section{HAL Id: hal-01071013 https://hal.science/hal-01071013}

Submitted on 7 Oct 2014

HAL is a multi-disciplinary open access archive for the deposit and dissemination of scientific research documents, whether they are published or not. The documents may come from teaching and research institutions in France or abroad, or from public or private research centers.
L'archive ouverte pluridisciplinaire HAL, est destinée au dépôt et à la diffusion de documents scientifiques de niveau recherche, publiés ou non, émanant des établissements d'enseignement et de recherche français ou étrangers, des laboratoires publics ou privés. 


\title{
Best decay rate, observability and open-loop admissibility costs: discussions and numerical study
}

\author{
Kaïs AMMARI * and Arnaud MÜNCH ${ }^{\dagger}$
}

\begin{abstract}
We show that the best decay rate can be estimated by the observability (or controllability) cost and open-loop admissibility cost. Moreover, we propose a numerical strategy to give an estimation for the best decay rate for a large class of evolution systems. Some examples are given to illustrate this new method.
\end{abstract}

AMS subject classification (2010): 35B35, 35B40, 35F05, 37L15.

Key words and phrases: best decay rate, observability cost, open-loop admissibility cost, numerical study.

\section{Introduction and main results}

Let $H$ be a Hilbert space equipped with the norm $\|.\|_{H}$, and let $A: \mathcal{D}(A) \subset H \rightarrow H$ be self-adjoint, positive and with boundedly invertible operator. We introduce the scale of Hilbert spaces $H_{\beta}, \beta \in \mathbb{R}$, as follows: for every $\beta \geq 0, H_{\beta}=\mathcal{D}\left(A^{\beta}\right)$, with the norm $\|z\|_{\beta}=\left\|A^{\beta} z\right\|_{H}$. The space $H_{-\beta}$ is defined by duality with respect to the pivot space $H$ as follows: $H_{-\beta}=H_{\beta}^{*}$ for $\beta>0$. The operator $A$ can be extended (or restricted) to each $H_{\beta}$, such that it becomes a bounded operator

$$
A: H_{\beta} \rightarrow H_{\beta-1} \quad \forall \beta \in \mathbb{R} .
$$

Let a bounded linear operator $B: U \rightarrow H_{-\frac{1}{2}}$, where $U$ is another Hilbert space which will be identified with its dual.

The systems we consider are described by

$$
\begin{aligned}
& \ddot{x}(t)+A x(t)=B v(t), \\
& x(0)=x_{0}, \dot{x}(0)=x_{1},
\end{aligned}
$$

and

$$
\begin{gathered}
\ddot{x}(t)+A x(t)+B B^{*} \dot{x}(t)=0, \\
x(0)=x_{0}, \dot{x}(0)=x_{1},
\end{gathered}
$$

where $t \in[0, \infty)$ is the time and $v \in L_{\text {loc }}^{2}\left(\mathbb{R}^{+}, U\right)$ which denoted the control function.

\footnotetext{
${ }^{*}$ UR Analyse et Contrôle des Edp, UR 13E64, Département de Mathématiques, Faculté des Sciences de Monastir, Université de Monastir, 5019 Monastir, Tunisie, e-mail: kais.ammari@fsm.rnu.tn

†Laboratoire de Mathématiques, UFR Sciences - Campus des Cezeaux, 63177 Aubière cedex, France, e-mail: arnaud.munch@math.univ-bpclermont.fr
} 
We can rewrite the system (1.2) as a first order differential equation, by putting $z(t)=$ $\left(\begin{array}{c}x(t) \\ \dot{x}(t)\end{array}\right):$

$$
\dot{z}(t)+\mathcal{A} z(t)=\mathcal{B} v(t), z(0)=z_{0}=\left(\begin{array}{c}
x_{0} \\
x_{1}
\end{array}\right)
$$

where

$$
\begin{gathered}
\mathcal{A}=\left(\begin{array}{cc}
0 & -I \\
A & 0
\end{array}\right): \mathcal{D}(\mathcal{A})=H_{1} \times H_{\frac{1}{2}} \subset \mathcal{H}=H_{\frac{1}{2}} \times H \rightarrow \mathcal{H}, \mathcal{B}=\left(\begin{array}{c}
0 \\
B
\end{array}\right) \in \mathcal{L}\left(U, \mathcal{H}_{-1}\right), \\
\mathcal{H}_{-1}=H \times H_{-\frac{1}{2}} .
\end{gathered}
$$

By the same way the system (1.4)-(1.5) can be also rewritten by :

$$
\dot{z}(t)+\mathcal{A}_{d} z(t)=0, z(0)=z_{0},
$$

where

$$
\mathcal{A}_{d}=\mathcal{A}+\mathcal{B B}^{*}: \mathcal{D}\left(\mathcal{A}_{d}\right)=\left\{\left(\begin{array}{c}
z_{1} \\
z_{2}
\end{array}\right) \in H_{\frac{1}{2}} \times H_{\frac{1}{2}}, A z_{1}+B B^{*} z_{2} \in H\right\} \subset \mathcal{H} \rightarrow \mathcal{H}
$$

It is clear that the operator $\mathcal{A}$ is skew-adjoint on $\mathcal{H}$ and hence, it generates a strongly continous group of unitary operators on $\mathcal{H}$, denoted by $(\mathcal{S}(t))_{t \in \mathbb{R}}$ and have a natural extensions to the Hilbert space $\mathcal{H}_{-1}$.

It is easy to see that, if $z_{0} \in \mathcal{H}$ and $u \in L_{l o c}^{2}\left(\mathbb{R}^{+}, U\right)$, then $z$ is $C\left([0, T], \mathcal{H}_{-1}\right), \forall T>0$ and given by

$$
z(t)=\mathcal{S}(t) z_{0}+\int_{0}^{t} \mathcal{S}(t-s) \mathcal{B} v(s) d s .
$$

If $\mathcal{B}$ were an admissible control operator for $(\mathcal{S}(t))_{t \in \mathbb{R}}{ }^{*}$, then $z \in C([0, T], \mathcal{H}), \forall T>0$.

Since $\mathcal{A}_{d}$ is dissipative and onto, it generates a contraction semigroup on $\mathcal{H}$, denoted by $\left(\mathcal{S}_{d}(t)\right)_{t \in \mathbb{R}^{+}}$.

The system (1.4)-(1.5) is well-posed. More precisely, the following classical result, holds.

Proposition 1.1. Suppose that $\left(x_{0}, x_{1}\right) \in \mathcal{H}$. Then the problem (1.4)-(1.5) admits a unique solution

$$
(x, \dot{x}) \in C([0, \infty) ; \mathcal{H}) .
$$

Moreover $(x(t), \dot{x}(t))$ satisfies, for all $t \geq 0$, the energy estimate

$$
E(0)-E(t)=\int_{0}^{t}\left\|B^{*} \dot{x}(s)\right\|_{U}^{2} \mathrm{~d} s,
$$

where $E(t)=\frac{1}{2}\|(x(t), \dot{x}(t))\|_{\mathcal{H}}^{2}$.

${ }^{*} \mathcal{B}$ is an admissible control operator for $(\mathcal{S}(t))_{t \in \mathbb{R}}$ means that : if for all $T>0$ there exists a constant $C>0$ such that for all $z_{0} \in \mathcal{H}_{1}=H_{1} \times H_{\frac{1}{2}}$, we have:

$$
\int_{0}^{T}\left\|\mathcal{B}^{*} \mathcal{S}(t) z_{0}\right\|_{U}^{2} d t \leq C\left\|z_{0}\right\|_{\mathcal{H}}^{2}
$$


From (1.8) it follows that the mapping $t \mapsto\|(x(t), \dot{x}(t))\|_{\mathcal{H}}^{2}$ is non increasing. In many applications it is important to know if this mapping decays exponentially when $t \rightarrow \infty$, i.e. if the system (1.4)-(1.5) is exponentially stable. One of the methods currently used for proving such exponential stability results is based on an observability inequality for the undamped system associated to the initial value problem

$$
\begin{gathered}
\ddot{\phi}(t)+A \phi(t)=0, \\
\phi(0)=x_{0}, \dot{\phi}(0)=x_{1} .
\end{gathered}
$$

It is well known that (1.9)-(1.10) is well-posed in $\mathcal{H}_{1}=H_{1} \times H_{\frac{1}{2}}$ and in $\mathcal{H}$. The result below, proved in [4], shows that the exponential stability of (1.4)-(1.5) is equivalent to an observability inequality for (1.9)-(1.10).

Theorem 1.2 (Ammari-Tucsnak [5]). Suppose that for all $\alpha>0$ fixed,

$$
\text { (H) } \sup _{\Re s=\alpha}\|H(s)\|_{\mathcal{L}(U)}<\infty,
$$

where $H(s)=B^{*} s\left(s^{2} I+A\right)^{-1} B \in \mathcal{L}(U), \forall \Re s>0$.

Then, the system described by (1.4)-(1.5) is exponentially stable in $\mathcal{H}$ if and only if there exists $T, C_{T}>0$ such that

$$
C_{T} \int_{0}^{T}\left\|\mathcal{B}^{*} \mathcal{S}(t) z_{0}\right\|_{U}^{2} \mathrm{~d} t \geq\left\|z_{0}\right\|_{\mathcal{H}}^{2} \quad \forall z_{0} \in \mathcal{H}_{1}
$$

In this paper we study the best decay rate of the damped evolution system (1.4)-(1.5).

In order to state our result, we define the best decay rate, depending on $B$, as

$$
\begin{gathered}
\omega(B)=\inf \left\{\omega \mid \text { there exists } C=C(\omega)>0 \text { such that } E(t) \leq C(\omega) e^{2 \omega t} E(0),\right. \\
\text { for every solution of (1.4)-(1.5) with initial data in } \mathcal{H}\} .
\end{gathered}
$$

Let us now recall the following related properties:

- System (1.6) is exactly controllable in time $T$ if for all $z_{0} \in \mathcal{H}_{-1}$, there is a $v \in L^{2}([0, T], U)$, $v(t)=0 \forall t \notin[0, T]$, such that $z(T)=0$. The controllability cost for (1.6) in time $T$ is the smallest positive constant $k_{T}$ in the following inequality for all such $v$ and $z_{0}$ :

$$
\int_{0}^{T}\|v(t)\|_{U}^{2} d t \leq k_{T}\left\|z_{0}\right\|_{\mathcal{H}_{-1}}^{2} .
$$

The observability cost for (1.9)-(1.10) in time $T$, is the smallest positive constant $C_{T}$ such that the inequality (1.12) is satisfied, and is equal to the controllability cost for system (1.6) in time $T$.

- On the other hand, the open-loop system (1.6), with $z_{0}=0$, is admissible for $(\mathcal{S}(t))_{t \in \mathbb{R}}$ if for all $T>0$ there exists a constant $C>0$ such that for all $v \in L^{2}(0, T, U)$ we have

$$
\int_{0}^{T}\left\|\mathcal{B}^{*} z(t)\right\|_{U}^{2} d t \leq C\|v\|_{L^{2}(0, T, U)}^{2} .
$$

The open-loop admissibility cost, denoted by $\widetilde{k_{T}}$, for (1.6), with $z_{0}=0$, in time $T$, is the smallest positive constant $C$ such that the inequality (1.15) is satisfied. 
The idea now is to use the observability (means an optimal-observability) to give an estimation of the best decay rate.

The problem of finding the optimal decay rate is difficult and has not a complete answer in the general case. We refer to [4], [6], [12], [14], [9] and to references therein. The main novelties brought in by this paper is that, according to Theorem 1.2, to propose a theoretical and numerical method to give a good estimation for the best decay rate for a large class of evolution systems in function of the observability and open-loop admissibility costs. In other words, we give a relation between the (best)-exact observability and the best decay rate.

Our main results, on the estimation of the best decay rate, are

Theorem 1.3. If the assumption (1.11) and observability inequality (1.12) are hold then,

1.

$$
\omega(B) \leq \inf _{\alpha>0, T_{o b s}>0}\left[\frac{1}{2 T} \ln \left(1-\frac{1}{k_{T}\left[1+\left(\sup _{\Re \lambda=\alpha}\left\|B^{*} \lambda\left(\lambda^{2} I+A\right)^{-1} B\right\|_{\mathcal{L}(U)}\right)^{2}\right]}\right)\right]<0,
$$

where $T_{\text {obs }}>0$ is such that the inequality (1.12) is holds.

2.

$$
\omega(B) \leq \inf _{T \geq T_{o p}}\left[\frac{1}{2 T} \ln \left(1-\frac{1}{k_{T}\left(\widetilde{k_{T}}+1\right)}\right)\right]<0,
$$

where $T_{o p}>0$ is the smallest time such that the inequality (1.12) holds.

In other words, if all finite energy solutions of (1.4)-(1.5) are exponentially stable then the best decay rate of the solutions of (1.4)-(1.5) satisfies (1.16) and (1.17).

Remark 1.4. The inequalities (1.16) and (1.17) are equivalent, see the proof. But, for the applications, we can use the first or the second one in function of the complexity of the case where we can compute $k_{T}, \widetilde{k_{T}}$ or the transfer function $H(\lambda)$ defined in Theorem 1.2.

The paper is organized as follows. Section 2 contains some background, on control, observability and stabilization, needed in the following sections. Section 3 is devoted to the proof of Theorem 1.3. Some applications and numerical study are given in Sections 4 and 5 .

\section{Some background on observability and on stability (see $[1,5])$}

Consider the evolution problem

$$
\begin{gathered}
\ddot{y}(t)+A y(t)=B v(t), \\
y(0)=\dot{y}(0)=0 .
\end{gathered}
$$

A natural question is the regularity of $y$ when $v \in L^{2}(0, T ; U)$. By applying standard energy estimates we can easily check that $y \in C(0, T ; H) \cap C^{1}\left(0, T ; H_{-\frac{1}{2}}\right)$. However if $B$ satisfies a certain admissibility condition then $y$ is more regular. More precisely the following result, which is a version of the general transposition method, holds true. 
Lemma 2.1. Suppose that $v \in L^{2}(0, T ; U)$ and that the solutions $\phi$ of (1.9)-(1.10) are such that $B^{*} \phi(.) \in H^{1}(0, T ; U)$ and there exists a constant $C>0$ such that

$$
\left\|\left(B^{*} \dot{\phi}\right)(\cdot)\right\|_{L^{2}(0, T ; U)} \leq C\left\|\left(x_{0}, x_{1}\right)\right\|_{\mathcal{H},} \quad \forall\left(x_{0}, x_{1}\right) \in \mathcal{H} .
$$

Then the problem (2.1)-(2.2) admits a unique solution having the regularity

$$
y \in C\left(0, T ; H_{\frac{1}{2}}\right) \cap C^{1}(0, T ; H) .
$$

Proof. Let

$$
\mathcal{D}(\mathcal{A})=\mathcal{H}_{1}
$$

and denote by $\mathcal{H}_{-1}$ the dual space of $\mathcal{H}_{1}$ with respect to the pivot space $\mathcal{H}$.

If we put $Z=\left(\begin{array}{c}y \\ \dot{y}\end{array}\right)$ it is clear that (2.1)-(2.2) can be written as

$$
\dot{Z}+\mathcal{A} Z(t)=\mathcal{B} v(t), Z(0)=0
$$

where

$$
\begin{gathered}
\mathcal{A}=\left(\begin{array}{cc}
0 & -I \\
A & 0
\end{array}\right): \mathcal{H} \subset \mathcal{H}_{-1} \rightarrow \mathcal{H}_{-1}, \\
\mathcal{B}=\left(\begin{array}{c}
0 \\
B
\end{array}\right): U \rightarrow \mathcal{H}_{-1} .
\end{gathered}
$$

It is well known that $\mathcal{A}$ is a skew adjoint operator so it generates a group of isometries in $\mathcal{H}_{-1}$, denoted by $\mathcal{S}(t)$.

After simple calculations we get that the operator $\mathcal{B}^{*}: \mathcal{H}_{1} \rightarrow U$ is given by

$$
\mathcal{B}^{*}\left(\begin{array}{l}
u \\
v
\end{array}\right)=B^{*} v, \forall(u, v) \in \mathcal{D}(\mathcal{A})
$$

This implies that

$$
\mathcal{B}^{*} \mathcal{S}^{*}(t)\left(\begin{array}{c}
x_{0} \\
x_{1}
\end{array}\right)=B^{*} \dot{\phi}, \forall\left(x_{0}, x_{1}\right) \in \mathcal{D}(\mathcal{A}),
$$

with $\phi$ satisfying (1.9)-(1.10). From the inequality above and (2.3) we deduce that there exists a constant $C>0$ such that

$$
\int_{0}^{T}\left\|\mathcal{B}^{*} \mathcal{S}^{*}(t)\left(\begin{array}{c}
x_{0} \\
x_{1}
\end{array}\right)\right\|_{U}^{2} d t \leq C\left\|\left(x_{0}, x_{1}\right)\right\|_{\mathcal{H}}^{2}, \forall\left(x_{0}, x_{1}\right) \in \mathcal{D}(\mathcal{A}) .
$$

According to Theorem 3.1 in $[8$, p.187] the inequality above implies the interior regularity (2.4).

Proposition 2.2. Suppose that $v \in L^{2}(0, T ; U)$ and that the problem (2.1)-(2.2) admits a unique solution having the regularity

$$
y \in C\left(0, T ; H_{\frac{1}{2}}\right) \cap C^{1}(0, T ; H) .
$$

Then hypothesis $(H)$ holds if and only if $B^{*} y(.) \in H^{1}(0, T ; U)$ and there exists a constant $C=\sup _{\Re s=\alpha}\|H(s)\|_{\mathcal{L}(U)}>0$ such that

$$
\left\|B^{*} \dot{y}(\cdot)\right\|_{L^{2}(0, T ; U)} \leq C\|v\|_{L^{2}(0, T ; U)}, \quad \forall v \in L^{2}(0, T ; U) .
$$


Proof. As equation (2.1) is time reversible, after extending $v$ by zero for $t \in \mathbb{R} \backslash[0, T]$, we can solve (2.1)-(2.2), for $t \in \mathbb{R}$. By this way, we obtain a function, denoted also by $y$, such that

$$
\begin{aligned}
& y \in C\left(\mathbb{R} ; H_{\frac{1}{2}}\right) \cap C^{1}(\mathbb{R} ; H) \cap L^{2}\left(\mathbb{R} ; H_{\frac{1}{2}}\right), \\
& y(t)=0, \quad \forall t \leq 0,
\end{aligned}
$$

and $y$ satisfies (2.1)-(2.2) for all $t \in \mathbb{R}$.

Let $\widehat{y}(\lambda)$, where $\lambda=\gamma+i \eta, \gamma>0$ and $\eta \in \mathbb{R}$, be the Laplace (with respect to t) transform of $y$. Since $y$ satisfies (2.7), estimate (2.6) is equivalent to the fact that the function $t \rightarrow e^{-\gamma t} B^{*} y(t)$ belongs to $H^{1}(\mathbb{R} ; U)$ and that there exists a constant $M_{1}>0$ such that

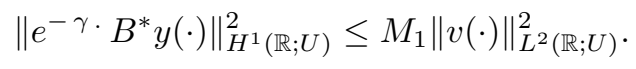

Equivalently, by the Parseval identity (see for instance Doetsch [13, p.212]), it suffices to prove that the function

$$
\eta \rightarrow(\gamma+i \eta) B^{*} \widehat{y}(\gamma+i \eta)
$$

belongs to $L^{2}\left(\mathbb{R}_{\eta} ; U\right)$, for some $\gamma>0$, and that there exists a constant $M_{2}>0$ such that

$$
\left\|(\gamma+i \eta) B^{*} \widehat{y}(\gamma+i \eta)\right\|_{L^{2}\left(\mathbb{R}_{\eta} ; U\right)}^{2} \leq M_{2} \int_{-\infty}^{+\infty}\|\widehat{v}(\gamma+i \eta)\|_{U}^{2} d \eta
$$

It can be easily checked that $\widehat{y}$ satisfies :

$$
\lambda^{2} \widehat{y}(\lambda)+A \widehat{y}(\lambda)=B \widehat{v}(\lambda), \forall \operatorname{Re} \lambda>0
$$

Relation above implies, for $\operatorname{Re} \lambda>0$ that

$$
\lambda B^{*} \widehat{y}(\lambda)=H(\lambda) \widehat{v}(\lambda), \forall \operatorname{Re} \lambda>0,
$$

where $H(\lambda)$ is defined in (1.11). Assumption (H) implies the existence of a constant $M_{2}>0$ such that (2.8) holds true. This ends the proof of the fact that assumption $(\mathrm{H})$ implies that (2.6) holds for all finite energy solution of (2.1)-(2.2).

Suppose now that (2.6) holds true. By using the time reversibility and the invariance with respect to translations (in time) of (2.1) we obtain that (2.1)-(2.2) is well posed for all input $v \in L^{2}(\mathbb{R}, U), v$ compactly supported. More precisely, we have

$$
\int_{\text {supp }(v)} \|\left(B^{*} \dot{y}(t)\left\|_{U}^{2} d t \leq C \int_{\operatorname{supp}(v)}\right\| v(t) \|_{U}^{2} d t\right.
$$

for all compactly supported $v \in L^{2}(\mathbb{R}, U)$, with the same constant as in (2.6).

Using (2.10) it follows that

$$
\|H(\gamma+i \eta) \widehat{v}(\gamma+i \eta)\|_{L^{2}\left(\mathbb{R}_{\eta}, U\right)}^{2} \leq C\|\widehat{v}(\lambda)\|_{L^{2}\left(\mathbb{R}_{\eta}, U\right)}^{2},
$$

for all compactly supported $v \in L^{2}(\mathbb{R}, U)$.

By density it follows that (2.11) holds for all $v \in L^{2}(\mathbb{R}, U)$. We have thus proved that (2.6) implies that $(\mathrm{H})$ holds true.

Proposition 2.3. Suppose that hypothesis $(H)$ is satisfied. Then for $\left(x_{0}, x_{1}\right) \in H_{\frac{1}{2}} \times H$ we have that $B^{*} \phi(.) \in H^{1}(0, T ; U)$ and there exist $C, T>0$ such that the solution $\phi(t)$ of $(1.9)-(1.10)$ satisfies (2.3). In the other words assumption (H) implies (2.3). 
Proof. Suppose that hypothesis $(\mathrm{H})$ is satisfied. Let $x(t) \in C\left(0, T ; H_{\frac{1}{2}}\right) \cap C^{1}(0, T ; H)$ be the unique solution of (1.4)-(1.5). By Proposition 1.1 we know that $B^{*} x \in H^{1}(0, T ; U)$ and that (1.8) holds true. Let $\phi$ be the solution of (1.9)-(1.10). We clearly have $\psi=x-\phi \in C\left(0, T ; H_{\frac{1}{2}}\right) \cap$ $C^{1}(0, T ; H)$ and $\psi$ satisfies

$$
\left\{\begin{array}{l}
\ddot{\psi}(t)+A \psi(t)=B B^{*} \dot{x}(t), \text { in } C\left(0, T ; H_{-\frac{1}{2}}\right), \\
\psi(0)=\dot{\psi}(0)=0 .
\end{array}\right.
$$

By applying now Proposition 2.2 with $v=B^{*} \dot{x} \in L^{2}(0, T ; U)$ we obtain that

$$
\int_{0}^{T}\left\|B^{*} \dot{\psi}(t)\right\|_{U}^{2} d t \leq C \int_{0}^{T}\left\|B^{*} \dot{x}(t)\right\|_{U}^{2} d t .
$$

Since $B^{*} \phi=B^{*} x-B^{*} \psi$ relations (1.8) and (2.12) imply the conclusion of the proposition.

Corollary 2.4. Suppose that assumption $(H)$ is satisfied. Then, for all $v \in L^{2}(0, T ; U),(2.1)$ (2.2) admits a unique solution y satisfying (2.5) and (2.6).

Proof. Suppose that assumption (H) is satisfied. Then Proposition 2.3 and Lemma 2.1 imply that problem (2.1)-(2.2) admits a unique solution $y$ satisfying (2.5). Finally Proposition 2.2 implies that $y$ satisfies (2.6). as

Let $x(t) \in C\left(0, T ; H_{\frac{1}{2}}\right) \cap C^{1}(0, T ; H)$ be the solution of $(1.4)-(1.5)$. Then $x(t)$ can be written

$$
x(t)=\phi(t)+\psi(t),
$$

where $\phi(t)$ satisfies (1.9)-(1.10) and $\psi(t)$ satisfies

$$
\begin{gathered}
\ddot{\psi}(t)+A \psi(t)=-B B^{*} \dot{x}(t), \\
\psi(0)=\dot{\psi}(0)=0 .
\end{gathered}
$$

\section{Proof of Theorem 1.3}

The main ingredient of the main results is the following result.

Lemma 3.1. Let $\left(x_{0}, x_{1}\right) \in \mathcal{H}$ and suppose that $(H)$ is verified. Then the solution $x(t)$ of (1.4)-(1.5) and the solution $\phi(t)$ of (1.9)-(1.10) satisfy

1.

$$
\begin{aligned}
\frac{1}{2\left[1+\left(\sup _{\Re s=\alpha}\|H(s)\|_{\mathcal{L}(U)}\right)^{2}\right]} \int_{0}^{T}\left\|B^{*} \dot{\phi}(t)\right\|_{U}^{2} d t \\
\leq \int_{0}^{T}\left\|B^{*} \dot{x}(t)\right\|_{U}^{2} d t \leq 4 \int_{0}^{T}\left\|B^{*} \dot{\phi}(t)\right\|_{U}^{2} d t .
\end{aligned}
$$

2.

$$
\frac{1}{2\left(1+\widetilde{k_{T}}\right)} \int_{0}^{T}\left\|B^{*} \dot{\phi}(t)\right\|_{U}^{2} d t \leq \int_{0}^{T}\left\|B^{*} \dot{x}(t)\right\|_{U}^{2} d t \leq 4 \int_{0}^{T}\left\|B^{*} \dot{\phi}(t)\right\|_{U}^{2} d t
$$


Remark 3.2. By Proposition $1.1, B^{*} \dot{x}(\cdot) \in L^{2}(0, T ; U)$. So, equation (2.14) makes sense. The result above shows that the $L^{2}$ norm of $\left\|B^{*} \dot{x}(\cdot)\right\|_{U}$ is equivalent to the $L^{2}$ norm of $\left\|B^{*} \dot{\phi}(\cdot)\right\|_{U}$ (notice that $\left\|B^{*} \dot{\phi}(\cdot)\right\|_{U} \in L^{2}(0, T)$ by Proposition 2.3$)$.

Proof of Lemma 3.1. We prove (3.16) for $x(t)$ satisfying (1.4)-(1.5) and $\phi(t)$ solution of (1.9)(1.10).

Relation (2.13) implies that

$$
\int_{0}^{T}\left\|B^{*} \dot{\phi}(\cdot)\right\|_{U}^{2} d t \leq 2\left\{\int_{0}^{T}\left\|B^{*} \dot{x}(\cdot)\right\|_{U}^{2} d t+\int_{0}^{T}\left\|B^{*} \dot{\psi}(\cdot)\right\|_{U}^{2} d t\right\} .
$$

Estimate above combined with inequality (2.6) in Proposition 2.2 implies that

$$
\frac{1}{2\left[1+\left(\sup _{\Re s=\alpha}\|H(s)\|_{\mathcal{L}(U)}\right)^{2}\right]} \int_{0}^{T} \|\left(B ^ { * } \dot { \phi } ( \cdot ) \| _ { U } ^ { 2 } d t \leq \int _ { 0 } ^ { T } \| \left(B^{*} \dot{x}(\cdot) \|_{U}^{2} d t .\right.\right.
$$

On the other hand, according to Remark 3.2 and to relation (2.13) we have that

$$
\left\|B^{*} \dot{\phi}(\cdot)\right\|_{U} \in L^{2}(0, T)
$$

This means that (2.14) can be rewritten as

$$
\ddot{\psi}(t)+A \psi(t)+B B^{*} \dot{\psi}(t)=-B B^{*} \dot{\phi}(t) .
$$

We denote now by $w(t)$ the extension of $B^{*} \dot{\phi}$ obtained by defining $w(t)=0, t \in \mathbb{R} \backslash[0, T]$. We still denote by $\psi(t)$ the solution of

$$
\left\{\begin{array}{l}
\ddot{\psi}(t)+A \psi(t)+B B^{*} \dot{\psi}(t)=-B w(t), t \in \mathbb{R} \\
\psi(0)=\dot{\psi}(0)=0
\end{array}\right.
$$

We clearly have $\psi(t)=0$ for $t \in \mathbb{R} \backslash[0, T]$.

Taking the Laplace transform we get

$$
\lambda^{2} \widehat{\psi}(\lambda)+A \widehat{\psi}(\lambda)+\lambda B B^{*} \widehat{\psi}(\lambda)=-B \widehat{w}(\lambda), \forall \lambda=\gamma+i \eta, \gamma>0 .
$$

The equality above holds in $H_{-\frac{1}{2}}$.

By applying $\bar{\lambda} \bar{\psi} \in H_{\frac{1}{2}}$ to the equality above, we get

$$
\lambda|\lambda|^{2}\|\widehat{\psi}(\lambda)\|_{X}^{2}+\bar{\lambda}\left\|A^{\frac{1}{2}} \widehat{\psi}(\lambda)\right\|_{X}^{2}+\left\|\lambda B^{*} \widehat{\psi}(\lambda)\right\|_{U}^{2}=-<w(\lambda), \bar{\lambda} B^{*} \overline{\widehat{\psi}}(\lambda)>_{U} .
$$

Taking the real part of each term, we get

$$
\int_{\mathbb{R}_{\eta}}\left\|\lambda B^{*} \widehat{\psi}(\lambda)\right\|_{U}^{2} d \eta \leq \frac{1}{2} \int_{\mathbb{R}_{\eta}}\|\widehat{w}(\lambda)\|_{U}^{2} d \eta+\frac{1}{2} \int_{\mathbb{R}_{\eta}}\left\|\lambda B^{*} \widehat{\psi}(\lambda)\right\|_{U}^{2} d \eta .
$$

Parseval identity implies

$$
\left\|B^{*} \dot{\psi}(t)\right\|_{L^{2}(0, T ; U)}^{2} \leq\left\|B^{*} \dot{\phi}(t)\right\|_{L^{2}(0, T ; U)}^{2} .
$$

Relation (2.13) and inequality above imply that

$$
\left\|B^{*} \dot{x}(t)\right\|_{L^{2}(0, T ; U)}^{2} \leq 4\left\|B^{*} \dot{\phi}(t)\right\|_{L^{2}(0, T ; U)}^{2} .
$$

Inequalities (3.18) and (3.22) obviously yield the conclusion (3.16).

By the same way we obtain (3.17). 
We can now prove the main results.

Proof of Theorem 1.3. All finite energy solutions of (1.4)-(1.5) satisfy the estimate

$$
E(t) \leq M e^{2 \omega t} E(0), \quad \forall t \geq 0,
$$

where $M,-\omega>0$ are constants independent of $\left(x_{0}, x_{1}\right)$, if and only if there exist a time $T>0$ and a constant $C>0$ (depending on $T$ ) such that

$$
E(0)-E(T) \geq C E(0), \quad \forall\left(x_{0}, x_{1}\right) \in \mathcal{H} .
$$

By (1.8) relation above is equivalent to the inequality

$$
\int_{0}^{T}\left\|B^{*} \dot{x}(s)\right\|_{U}^{2} d s \geq C E(0), \quad \forall\left(x_{0}, x_{1}\right) \in \mathcal{H}
$$

and $w=\frac{\ln \left(1-\frac{1}{C}\right)}{2 T}>0, M=\frac{C}{C-1}>0$.

From Lemma 3.1 it follows that the system (1.4)-(1.5) is exponentially stable if and only if

$$
\int_{0}^{T}\left\|B^{*} \dot{\phi}(s)\right\|_{U}^{2} d s \geq C E(0), \quad \forall\left(x_{0}, x_{1}\right) \in \mathcal{H}_{1}
$$

holds true. By density it follows that (1.4)-(1.5) is exponentially stable if and only if (1.12) holds true.

Thus, if the exact observability of the system (1.9)-(1.10) is satisfied, i.e.

$$
k_{T} \int_{0}^{T}\left\|\mathcal{B}^{*} \mathcal{S}(t) z_{0}\right\|_{U}^{2} d t \geq\left\|z_{0}\right\|_{\mathcal{H}}^{2}, \forall z_{0} \in \mathcal{H}_{1}
$$

then according to Lemma 3.1 it follows that

$$
\int_{0}^{T}\left\|B^{*} \dot{x}(s)\right\|_{U}^{2} d s \geq \frac{1}{k_{T}\left[1+\left(\sup _{\Re s=\alpha}\|H(s)\|_{\mathcal{L}(U)}\right)^{2}\right]} E(0),
$$

and

$$
\int_{0}^{T}\left\|B^{*} \dot{x}(s)\right\|_{U}^{2} d s \geq \frac{1}{k_{T}\left(1+\widetilde{k_{T}}\right)} E(0)
$$

This implies that

$$
\text { with } C=\frac{1}{k_{T}\left[1+\left(\sup _{\Re s=\alpha}\|H(s)\|_{\mathcal{L}(U)}\right)^{2}\right]} \text { respectively } \frac{1}{2 T}
$$

This ends up the proof of Theorem 1.3.

\section{Applications and numerical study}

We now give some applications and numerical validations of Theorem 1.3. 


\subsection{First example: Stabilization of a string}

We consider the following initial and boundary problem:

$$
\left\{\begin{array}{l}
\frac{\partial^{2} u}{\partial t^{2}}-\frac{\partial^{2} u}{\partial x^{2}}+\frac{\partial u}{\partial t}(\xi, t) \delta_{\xi}=0,(x, t) \in(0,1) \times(0,+\infty) \\
u(0, t)=0, \frac{\partial u}{\partial x}(1, t)=0, t \in(0,+\infty) \\
u(x, 0)=u^{0}(x), \frac{\partial u}{\partial t}(x, 0)=u^{1}(x), x \in(0,1)
\end{array}\right.
$$

where $\xi \in(0,1)$ and $\delta_{\xi}$ is the Dirac mass concentrated in the point $\xi \in(0,1)$.

In this case, we have:

$$
H=L^{2}(0,1), U=\mathbb{R}, H_{\frac{1}{2}}=\left\{u \in H^{1}(0,1), u(0)=0\right\}
$$

and

$$
A=-\frac{d^{2}}{d x^{2}}, \mathcal{D}(A)=\left\{u \in H^{2}(0,1) \cap H_{\frac{1}{2}}, \frac{d u}{d x}(1)=0\right\}, B k=k \delta_{\xi}, \forall k \in \mathbb{R} .
$$

Then, $\mathcal{A}_{d}$ is given by

$$
\begin{gathered}
\mathcal{A}_{d}\left(\begin{array}{c}
u \\
v
\end{array}\right)=\left(\begin{array}{c}
v \\
\frac{d^{2} u}{d x^{2}}-v(\xi) \delta_{\xi}
\end{array}\right), \\
\forall(u, v) \in \mathcal{D}\left(\mathcal{A}_{d}\right)=\left\{(u, v) \in\left[H_{\frac{1}{2}} \cap H^{2}(0, \xi) \cap H^{2}(\xi, 1)\right] \times H_{\frac{1}{2}}, \frac{d u}{d x}\left(\xi^{+}\right)-\frac{d u}{d x}\left(\xi^{-}\right)=v(\xi)\right\} .
\end{gathered}
$$

The system described by (4.1) is exponentially stable in $H_{\frac{1}{2}} \times L^{2}(0,1)$ if and only if $\xi \in(0,1)$ admits a co-prime factorization

$$
\xi=\frac{p}{q} \text { with p odd }
$$

In the case where $\xi=\frac{1}{2}$, the estimation of the best decay rate of (4.1) is then an immediate consequence of Theorem 1.3.

The following result holds true.

\section{Theorem 4.1 .}

$$
w\left(\delta_{\frac{1}{2}}\right) \leq \inf _{\alpha>0}\left[\frac{1}{4} \ln \left(1-\frac{4}{5+\frac{1}{s h^{2}\left(\frac{\alpha}{2}\right)}}\right)\right] .
$$

Remark 4.2. According to [4, Theorem 1.2], $w(B)=-\frac{1}{2} \ln (3) \approx-0.549306$. Therefore, Theorem 1.3 provides a rather satisfactory estimate of the best decay rate $w(B)$ since we compute that

$$
\inf _{\alpha} \frac{1}{4} \ln \left(1-\frac{4}{5+\frac{1}{s h^{2}\left(\frac{\alpha}{2}\right)}}\right)=-\frac{1}{4} \ln (5) \approx-0.402359 .
$$

The observability system associated to (4.1) reads as follows:

$$
\begin{gathered}
\frac{\partial^{2} \phi}{\partial t^{2}}-\frac{\partial^{2} \phi}{\partial x^{2}}=0,(0,1) \times(0,+\infty), \\
\phi(0, t)=0, \frac{\partial \phi}{\partial x}(1, t)=0,(0,+\infty), \\
\phi(x, 0)=u^{0}(x), \frac{\partial \phi}{\partial t}(x, 0)=u^{1}(x),(0,1) .
\end{gathered}
$$


Lemma 4.3. The operators $A$ and $B$ defined by (4.2) satisfy assumption (1.11).

Proof. Let $k \in \mathbb{R}$. It can be easily checked that $v=\left(\lambda^{2}+A\right)^{-1} B k$ satisfies:

$$
\begin{gathered}
\lambda^{2} v(x)-\frac{d^{2} v}{d x^{2}}(x)=0, x \in(0, \xi) \cup(\xi, 1), \operatorname{Re} \lambda>0, \\
v(0)=0, \frac{d v}{d x}(1)=0, \\
{[v]_{\xi}=0,\left[\frac{d v}{d x}\right]_{\xi}=k,}
\end{gathered}
$$

where we denote by $[g]$ the jump of the function $g$ at the point $\xi$.

The solutions of (4.8)-(4.9) have the form

$$
v(x)=\left\{\begin{array}{l}
A_{1} \operatorname{sh}(\lambda x), x \in(0, \xi), \\
A_{2} \operatorname{ch}[\lambda(x-1)], x \in(\xi, 1)
\end{array}\right.
$$

where $A_{1}, A_{2}$ are constants.

Consequently, the solutions of (4.8)-(4.10) have the following form

$$
v(x)=\left\{\begin{array}{l}
\frac{1}{\lambda} \frac{\operatorname{ch}[\lambda(\xi-1)] \operatorname{sh}(\lambda x)}{\operatorname{sh}(\lambda)} k, x \in(0, \xi), \\
\frac{1}{\lambda} \frac{\operatorname{sh}(\lambda \xi) \operatorname{ch}[(x-1)]}{\operatorname{sh}(\lambda)} k, x \in(\xi, 1),
\end{array}\right.
$$

Then, the function $H(\lambda)=\lambda B^{*}\left(\lambda^{2}+A\right)^{-1} B$ associated to problem (4.1) is given by the following expression

$$
H(\lambda)=\frac{\operatorname{sh}(\lambda \xi) \operatorname{ch}[\lambda(\xi-1)]}{\operatorname{ch}(\lambda)}, \operatorname{Re} \lambda>0 .
$$

We easily check that

$$
\sup _{\lambda \in \mathbb{C}_{\alpha}}|H(\lambda)| \leq \frac{\operatorname{ch}(\alpha \xi) \operatorname{ch}[\alpha(\xi-1)]}{\operatorname{sh}(\alpha)} .
$$

Thus the assumption $(\mathrm{H})$ is satisfied.

We remark that for $\xi=\frac{1}{2}$ we have that

$$
\sup _{\lambda \in \mathbb{C}_{\alpha}}|H(\lambda)|^{2} \leq \frac{1}{4}\left(1+\frac{1}{s h^{2}\left(\frac{\alpha}{2}\right)}\right)
$$

The observability inequality concerning the trace at the point $x=\xi$ of the solutions of (4.5)-(4.7) is given in the proposition below.

Proposition 4.4. Let $T \geq 2$ be fixed. Then the following assertion hold true.

For all $\xi \in(0,1)$ satisfying $(4.3)$ the solution $\phi$ of (4.5)-(4.7) satisfies

$$
\int_{0}^{T}\left[\frac{\partial \phi}{\partial t}(\xi, t)\right]^{2} d t \geq C_{\xi, T}\left\|\left(u^{0}, u^{1}\right)\right\|_{H_{\frac{1}{2}} \times L^{2}(0,1)}^{2}, \quad \forall\left(u^{0}, u^{1}\right) \in H_{\frac{1}{2}} \times L^{2}(0,1),
$$

where $C_{\xi, T}>0$ is a constant depending only on $\xi$ and $T$. 
Proof. If we put

$$
u^{0}(x)=\sum_{n=0}^{\infty} a_{n} \sin \left(\left(n+\frac{1}{2}\right) \pi x\right), u^{1}(x)=\sum_{n=0}^{\infty} b_{n} \sin \left(\left(n+\frac{1}{2}\right) \pi x\right)
$$

with

$$
\left(\left(n+\frac{1}{2}\right) \pi a_{n}\right),\left(b_{n}\right) \subset l^{2}(\mathbb{R})
$$

then we clearly have

$$
\frac{\partial \phi}{\partial t}(\xi, t)=\sum_{n \geq 0}\left(-\left(n+\frac{1}{2}\right) \pi a_{n} \sin \left(\left(n+\frac{1}{2}\right) \pi t\right)+b_{n} \cos \left(\left(n+\frac{1}{2}\right) \pi t\right)\right) \sin \left(\left(n+\frac{1}{2}\right) \pi \xi\right) .
$$

From Ingham's inequality [17] we obtain, for all $T \geq 2$, the existence of a constant $C_{T}>0$ such that the solution $\phi$ of (4.5)-(4.7) satisfies

$$
\int_{0}^{T}\left[\frac{\partial \phi}{\partial t}(\xi, t)\right]^{2} d t \geq C_{T} \sum_{n \geq 0}\left(\left(n+\frac{1}{2}\right)^{2} \pi^{2} a_{n}^{2}+b_{n}^{2}\right)\left|\sin \left(\left(n+\frac{1}{2}\right) \pi \xi\right)\right|^{2} .
$$

Relations (4.13) implies the existence of a constant $C_{\xi, T}>0$ such that

$$
\int_{0}^{T}\left[\frac{\partial \phi}{\partial t}(\xi, t)\right]^{2} d t \geq C_{T, \xi} \sum_{n=0}^{\infty}\left(\left(n+\frac{1}{2}\right)^{2} \pi^{2} a_{n}^{2}+b_{n}^{2}\right), \text { iff } \xi \in(0,1) \text { satisfied (4.3), }
$$

which is exactly (4.4).

We remark, according to the above proof, that in case where $\xi=\frac{1}{2}$ and $T=2$, we have

$$
\int_{0}^{2}\left[\frac{\partial \phi}{\partial t}\left(\frac{1}{2}, t\right)\right]^{2} d t=\sum_{n=0}^{\infty}\left(\left(n+\frac{1}{2}\right)^{2} \pi^{2} a_{n}^{2}+b_{n}^{2}\right)=\left\|\left(u^{0}, u^{1}\right)\right\|_{H_{\frac{1}{2}} \times L^{2}(0,1)}^{2},
$$

and the observability $\operatorname{cost} k_{T}=1$.

Proof of Theorem 4.1. According to Theorem 1.3,

$$
w\left(\delta_{\frac{1}{2}}\right) \leq \frac{1}{4} \ln \left(1-\frac{4}{5+\frac{1}{s^{2}\left(\frac{\alpha}{2}\right)}}\right), \quad \forall \alpha>0 .
$$

We end up in this way the proof of theorem.

\subsection{Second example: Interior stabilization of the wave equation}

\subsubsection{One-dimentional case}

We consider the following initial and boundary value problem:

$$
\begin{gathered}
\partial_{t}^{2} u(x, t)-\partial_{x}^{2} u(x, t)+2 a(x) \partial_{t} u(x, t)=0, \quad 0<x<1, t>0, \\
u(0, t)=u(1, t)=0, \quad t>0, \\
u(x, 0)=u^{0}(x), \quad \partial_{t} u(x, 0)=u^{1}(x), \quad 0<x<1,
\end{gathered}
$$


where $a \in L^{\infty}(0,1)$ is non-negative and satisfies that there exist an non empty open set $I$ of $(0,1)$ and a constant $c>0$ such that $a(x) \geq c$ for all $x \in I$. Here we assume that the string is of length 1.

In this case, we have:

$$
H=L^{2}(0,1), U=L^{2}(0,1), H_{\frac{1}{2}}=H_{0}^{1}(0,1)
$$

and

$$
A=-\frac{d^{2}}{d x^{2}}, \mathcal{D}(A)=H^{2}(0,1) \cap H_{0}^{1}(0,1), B=B^{*}=\sqrt{2 a} \in \mathcal{L}\left(L^{2}(0,1)\right) .
$$

If $u$ is a solution of (4.14)-(4.16), we define the energy of $u$ at instant $t$ by

$$
E(t)=\frac{1}{2} \int_{0}^{1}\left(\left(\partial_{t} u(x, t)\right)^{2}+\left(\partial_{x} u(x, t)\right)^{2}\right) d x .
$$

Simple formal calculations shows that a sufficiently smooth solution of (4.14)-(4.16) satisfies the energy estimate

$$
E(0)-E(t)=2 \int_{0}^{t} \int_{I} a(x)\left[\partial_{t} u(x, s)\right]^{2} d x d s, \quad \forall t \geq 0 .
$$

In particular, the previous estimate implies that $E(t) \leq E(0)$ for all $t \geq 0$. Moreover, estimate (4.19) suggests that the natural well-posedness space for (4.14)-(4.16) is $H_{0}^{1}(0,1) \times L^{2}(0,1)$. The inner product on $H_{0}^{1}(0,1) \times L^{2}(0,1)$ is defined by

$$
\langle[f, g],[u, v]\rangle:=\int_{0}^{1}\left(f^{\prime}(x) \overline{u^{\prime}(x)}+g(x) \overline{v(x)}\right) d x, \quad \text { for all }[f, g],[u, v] \text { in } H_{0}^{1}(0,1) \times L^{2}(0,1) \text {. }
$$

We have the following well-posedness result:

Proposition 4.5. Assume that $\left(u^{0}, u^{1}\right) \in H_{0}^{1}(0,1) \times L^{2}(0,1)$. Then the problem (4.14)-(4.16) admits a unique solution $u \in C\left(\left[0,+\infty\left[; H_{0}^{1}(0,1)\right) \cap C^{1}\left(\left[0,+\infty\left[; L^{2}(0,1)\right)\right.\right.\right.\right.$. Moreover u satisfies the energy estimate (4.19).

We define the best decay rate, as a function of $a$, as

$$
\begin{aligned}
& \omega(a)=\inf \left\{\omega ; \text { there exists } C=C(\omega)>0 \text { such that } E(t) \leq C(\omega) e^{2 \omega t} E(0),\right. \\
& \text { for every solution } \left.u \text { of }(4.14)-(4.16) \text { with initial data in } H_{0}^{1}(0,1) \times L^{2}(0,1)\right\} .
\end{aligned}
$$

According to (4.19), $w(a)<0$ for all nonnegative damped $a(x)$ which satisfies the above condition, see [16] and also [5].

By the same way as the first application we have the following

\section{Theorem 4.6.}

$$
w(a) \leq \inf _{T \geq 2} \frac{1}{2 T} \ln \left(1-\frac{1}{k_{T}\left(1+\widetilde{k_{T}}\right)}\right)<0,
$$

where $k_{T}$ and $\widetilde{k_{T}}$ are defined in Section 1 (see the following section for their numerical evaluation). 
We can obtain the same result for the Euler-Bernoulli beam equation, i.e.,

$$
\begin{gathered}
\partial_{t}^{2} u(x, t)+\partial_{x}^{4} u(x, t)+2 a(x) \partial_{t} u(x, t)=0, \quad 0<x<1, t>0, \\
u(0, t)=u(1, t)=0, \partial_{x}^{2} u(0, t)=\partial_{x}^{2} u(1, t)=0, \quad t>0, \\
u(x, 0)=u^{0}(x), \quad \partial_{t} u(x, 0)=u^{1}(x), \quad 0<x<1,
\end{gathered}
$$

where $a \in L^{\infty}(0,1)$ is non-negative and satisfies the same condition as above.

We can compare with the result obtained in [2] and [3] recently and Theorem 1.3. More precisely, according to [2] and [3], the best decay rate $w(a)$ is given by the spectral abscissa so we can compare numerically between the sepctral abscissa and the bound given by Theorem 1.3.

\subsubsection{High-dimentional case}

We consider the following initial and boundary value problems:

$$
\begin{gathered}
\partial_{t}^{2} u(x, t)-\Delta u(x, t)+2 a(x) \partial_{t} u(x, t)=0, \quad(x, t) \in \Omega \times(0,+\infty), \\
u=0, \quad \partial \Omega \times(0,+\infty), \\
u(x, 0)=u^{0}(x), \quad \partial_{t} u(x, 0)=u^{1}(x), \quad x \in \Omega,
\end{gathered}
$$

and

$$
\begin{gathered}
\partial_{t}^{2} u(x, t)+\Delta^{2} u(x, t)+2 a(x) \partial_{t} u(x, t)=0, \quad(x, t) \in \Omega \times(0,+\infty), \\
u=0, \quad \partial \Omega \times(0,+\infty), \\
u(x, 0)=u^{0}(x), \quad \partial_{t} u(x, 0)=u^{1}(x), \quad x \in \Omega,
\end{gathered}
$$

where $a \in L^{\infty}(\Omega)$ and satisfy some geometric control condition, see [18] for more details.

Here,

$$
H=L^{2}(\Omega), H_{\frac{1}{2}}=H_{0}^{1}(\Omega), U=L^{2}(\Omega)
$$

and

$$
A=-\Delta: \mathcal{D}(A)=H^{2}(\Omega) \cap H_{0}^{1}(\Omega) \subset L^{2}(\Omega) \rightarrow L^{2}(\Omega), B=B^{*}=\sqrt{2 a} \in \mathcal{L}\left(L^{2}(\Omega)\right) .
$$

According to [18] $w(a)$ is given by the minimum between a spectral abscissa of the dissipative operator and some geometrical quantity (which is linked to the Birkhoff limit of the damping coefficient $2 a$ under the bicaracteristics). We have the following estimation of $w(a)$.

$$
w(a) \leq \inf _{T \geq T_{o p}} \frac{1}{2 T} \ln \left(1-\frac{1}{k_{T}\left(1+\widetilde{k_{T}}\right)}\right)<0,
$$

where $k_{T}$ and $\widetilde{k_{T}}$ are defined in Section 1 (see the following section for their numerical evaluations).

\section{$5 \quad$ Numerical evaluation of the constants $k_{T}$ and $\widetilde{k_{T}}$}

We introduce in this section iterative methods to evaluate numerically, for any $T>0$ large enough, the two constants $k_{T}$ and $\widetilde{k_{T}}$ which appear in the inequality (1.17). 


\subsection{Evaluation of the constant $k_{T}$ : the internal damping case}

We consider the wave equation with the damping term:

$$
\left\{\begin{array}{l}
\partial_{t}^{2} u(x, t)-\Delta u(x, t)+2 a(x) \partial_{t} u(x, t)=0, \quad(x, t) \in \Omega \times(0, T):=Q_{T}, \\
u(0, t)=u(1, t)=0, \quad t \in(0, T), \\
u(x, 0)=u^{0}(x), \quad \partial_{t} u(x, 0)=u^{1}(x), \quad x \in \Omega .
\end{array}\right.
$$

We first discuss the evaluation of the constant $k_{T}$ which appears in the inequality:

$$
\left\|\varphi^{0}, \varphi^{1}\right\|_{H_{0}^{1}(\Omega) \times L^{2}(\Omega)}^{2} \leq k_{T} \iint_{Q_{T}} 2 a(x)\left|\varphi_{t}\right|^{2} d x d t
$$

where $\varphi$ solves the homogeneous equation

$$
\left\{\begin{array}{l}
\partial_{t}^{2} \varphi(x, t)-\Delta \varphi(x, t)=0, \quad(x, t) \in Q_{T} \\
\varphi(0, t)=\varphi(1, t)=0, \quad t \in(0, T) \\
\varphi(x, 0)=\varphi^{0}(x), \quad \partial_{t} \varphi(x, 0)=\varphi^{1}(x), \quad x \in \Omega
\end{array}\right.
$$

$k_{T}$ is an observability constant related to the wave equation and we may write

$$
k_{T}=\sup _{\left(\varphi^{0}, \varphi^{1}\right) \in H_{0}^{1}(\Omega) \times L^{2}(\Omega)} \frac{\left\|\varphi^{0}, \varphi^{1}\right\|_{H_{0}^{1}(\Omega) \times L^{2}(\Omega)}^{2}}{\iint_{Q_{T}} 2 a(x)\left|\varphi_{t}\right|^{2} d x d t} .
$$

and also, using by reducing the norm,

$$
k_{T}=\sup _{\left(\varphi^{0}, \varphi^{1}\right) \in L^{2}(\Omega) \times H^{-1}(\Omega)} \frac{\left\|\varphi^{0}, \varphi^{1}\right\|_{L^{2}(\Omega) \times H^{-1}(\Omega)}^{2}}{\iint_{Q_{T}} 2 a(x)|\varphi|^{2} d x d t} .
$$

For simple geometry, the constant $k_{T}$ may be evaluated easily using a Fourier analysis (we refer to the Appendix for the 1D case). In the general case, we may proceed as follows.

First, we define by $y$ the solution of the backward problem

$$
\left\{\begin{array}{lr}
L y=-a(x) \varphi & \text { in } Q_{T} \\
y=0 & \text { on } \Sigma_{T} \\
\left(y(\cdot, T), y_{t}(\cdot, T)\right)=(0,0) & \text { in } \Omega
\end{array}\right.
$$

and then the operator $\Lambda_{T}: L^{2}(\Omega) \times H^{-1}(\Omega) \rightarrow H_{0}^{1}(\Omega) \times L^{2}(\Omega)$ defined by $\Lambda_{T}\left(\varphi_{0}, \varphi_{1}\right):=$ $\left(y_{1},-y_{0}\right)$. The following equality

$$
\left(\Lambda_{T}\left(\varphi_{0}, \varphi_{1}\right),\left(\varphi_{0}, \varphi_{1}\right)\right)_{H_{0}^{1}(\Omega) \times L^{2}(\Omega), L^{2}(\Omega) \times H^{-1}(\Omega)}=\iint_{Q_{T}} a(x)|\varphi|^{2} d x d t
$$

allows to reformulate the inequality

$$
\left\|\varphi_{0}, \varphi_{1}\right\|_{L^{2} \times H^{-1}}^{2} \leq k_{T} \iint_{Q_{T}} a(x)|\varphi|^{2} d x d t
$$

as follows

$$
\left(\left(\varphi_{0}, \varphi_{1}\right),\left(\varphi_{0}, \varphi_{1}\right)\right)_{L^{2}(\Omega) \times H^{-1}(\Omega)} \leq k_{T}\left(\Lambda_{T}\left(\varphi_{0}, \varphi_{1}\right),\left(\varphi_{0}, \varphi_{1}\right)\right)_{H_{0}^{1}(\Omega) \times L^{2}(\Omega), L^{2}(\Omega) \times H^{-1}(\Omega)}
$$


for all $\left(\varphi_{0}, \varphi_{1}\right)$ in $L^{2}(\Omega) \times H^{-1}(\Omega)$. Recalling that the operator $\Lambda_{T}$ defines, for $T$ large enough, a symmetric, positive isomorphism from $L^{2}(\Omega) \times H^{-1}(\Omega)$ into $H_{0}^{1}(\Omega) \times L^{2}(\Omega)$ (see [19], chapter 7 ), the evaluation of the constant $k_{T}$ is reduced to the resolution of a generalized eigenvalue problem. Precisely, $\left(k_{T}\right)^{-1}$ coincides with the smallest eigenvalue of the operator $\Lambda_{T}$. In order to evaluate this smallest eigenvalue, we may use the inverse power iteration method (we refer to [10]) assuming that this eigenvalue is simple: in our context, the algorithm reads as follows:

Let $\left(\varphi_{0}^{0}, \varphi_{1}^{0}\right) \in L^{2}(\Omega) \times H^{-1}(\Omega)$ be any function; compute $\left(\psi_{0}^{0}, \psi_{1}^{0}\right) \in L^{2}(\Omega) \times H^{-1}(\Omega)$ defined by

$$
\left(\psi_{0}^{0}, \psi_{1}^{0}\right)=\frac{\left(\varphi_{0}^{0}, \varphi_{1}^{0}\right)}{\left\|\left(\varphi_{0}^{0}, \varphi_{1}^{0}\right)\right\|_{L^{2}(\Omega) \times H^{-1}(\Omega)}} .
$$

Then, for any $k \geq 1$, compute

$$
\left\{\begin{array}{l}
\left(\varphi_{0}^{k}, \varphi_{1}^{k}\right)=\Lambda_{T}^{-1}\left(\psi_{0}^{k-1},-\Delta^{-1} \psi_{1}^{k-1}\right), \\
\left(\psi_{0}^{k}, \psi_{1}^{k}\right)=\frac{\left(\varphi_{0}^{k}, \varphi_{1}^{k}\right)}{\left\|\left(\varphi_{0}^{k}, \varphi_{1}^{k}\right)\right\|_{L^{2}(\Omega) \times H^{-1}(\Omega)}}
\end{array}\right.
$$

If the smallest eigenvalue of the operator $\Lambda_{T}$ is isolated, then the sequences converge and the following property holds :

$$
\lim _{k \rightarrow \infty}\left(\left(\varphi_{0}^{k+1}, \varphi_{1}^{k+1}\right),\left(\psi_{0}^{k}, \psi_{1}^{k}\right)\right)_{L^{2}(\Omega) \times H^{-1}(\Omega), L^{2}(\Omega) \times H^{-1}(\Omega)}=\left(k_{T}\right)^{-1} .
$$

Remark 5.1. The first step of the algorithm requires to solve a null controllability problem: find $\left(\varphi_{0}^{k}, \varphi_{1}^{k}\right) \in L^{2} \times H^{-1}$ such that

$$
\Lambda_{T}\left(\varphi_{0}^{k}, \varphi_{1}^{k}\right)=\left(\psi_{0}^{k},-\Delta^{-1} \psi_{1}^{k}\right),
$$

that is, find the null control (of the form $-a(x) \varphi$ ) for $y$ solution of (5.35) with the initial data $\left(\Delta^{-1} \psi_{1}^{k}, \psi_{0}^{k}\right) \in H_{0}^{1}(\Omega) \times L^{2}(\Omega)$. We shall use the direct method developed in [11] which is very appropriate since it approximates the operator $\Lambda_{T}$ explicitly (for iterative indirect method, we refer to [15, 20]) and which ensures the strong convergence of the approximation.

\subsection{Evaluation of the constant $\widetilde{k_{T}}:$ the internal damping case}

The constant $\widetilde{k_{T}}$ which appears in the inequality

$$
\int_{Q_{T}} 2 a(x)\left|u_{t}\right|^{2} d x d t \leq \widetilde{k_{T}}\|v\|_{L^{2}\left(Q_{T}\right)}^{2}
$$

where $u$ solves

$$
\left\{\begin{array}{l}
\partial_{t}^{2} u(x, t)-\partial_{x}^{2} u(x, t)=\sqrt{2 a(x)} v, \quad(x, t) \in Q_{T} \\
u(0, t)=u(1, t)=0, \quad t \in(0, T), \\
u(x, 0)=0, \quad \partial_{t} u(x, 0)=0, \quad x \in \Omega
\end{array}\right.
$$

can be defined as :

$$
\widetilde{k_{T}}=\sup _{v \in L^{2}\left(Q_{T}\right)} \frac{\iint_{Q_{T}} 2 a(x)\left|u_{t}\right|^{2} d x d t}{\|v\|_{L^{2}\left(Q_{T}\right)}^{2}} .
$$

$\widetilde{k_{T}}$ is the constant of continuity of a linear map in $v$. Again, for simple geometry, we may employ Fourier decomposition. We refer to the Appendix for the 1D situation. As before, in the general case, we may proceed as follows. 
First, we introduce the function $\psi$ solution of

$$
\begin{cases}L \psi=-2 a(x) \frac{d}{d t}\left(u_{t}\right), & \text { in } Q_{T} \\ \psi=0, & \text { on } \Sigma_{T} \\ \psi(\cdot, T)=\psi_{t}(\cdot, T)=0 . & \end{cases}
$$

As in [19] (see page 416), the derivate is defined by duality so that

$$
<a(x) \frac{\partial}{\partial t}\left(\psi_{t}\right), w>=-\iint_{Q_{T}} a(x) \psi_{t} w_{t} d x d t, \quad \forall w \in H^{1}\left(0, T ; L^{2}(\Omega)\right) .
$$

The function $\psi$ is defined by transposition, so that in particular, we have

$$
\iint_{Q_{T}} 2 a(x)\left|u_{t}\right|^{2} d x d t=\iint_{Q_{T}} \sqrt{2 a(x)} \psi v d x d t
$$

Therefore, defining the operator $\mathcal{B}_{T}: L^{2}\left(Q_{T}\right) \rightarrow L^{2}\left(Q_{T}\right)$ by $\mathcal{B}_{T}(v):=\sqrt{2 a(x)} \psi$, we get

$$
\left(\mathcal{B}_{T} v, v\right)_{L^{2}\left(Q_{T}\right)} \leq \widetilde{k_{T}}(v, v)_{L^{2}\left(Q_{T}\right)}
$$

so that the constant $\widetilde{k_{T}}$ may again be approximated by the power iteration method, which reads as follows:

Let $v^{0}$ any function in $L^{2}\left(Q_{T}\right)$. We define $w^{0}=v^{0} /\left\|v^{0}\right\|_{L^{2}\left(Q_{T}\right)}$ in $L^{2}\left(Q_{T}\right)$ and then for any $k \geq 1$,

$$
\left\{\begin{array}{l}
v^{k}=\mathcal{B}_{T} w^{k-1} \\
w^{k}=v^{k} /\left\|v^{k}\right\|_{L^{2}\left(Q_{T}\right)}
\end{array}\right.
$$

Then, if the largest eigenvalue of $\mathcal{B}_{T}$ is isolated, the sequences $\left\{v^{k}\right\}_{k>0},\left\{w^{k}\right\}_{k>0}$ converge and

$$
\lim _{k \rightarrow \infty}\left(v^{k+1}, w^{k}\right)_{L^{2}\left(Q_{T}\right)}=\widetilde{k_{T}} .
$$

We easily check that the operator is symmetric: $\left(\mathcal{B}_{T} v, \bar{v}\right)=(v, \mathcal{B}(\bar{v}))$ for all $v, \bar{v} \in L^{2}\left(Q_{T}\right)$. Moreover, clearly $\left(\mathcal{B}_{T}(v), v\right) \geq 0$ for all $v \in L^{2}\left(Q_{T}\right)$. Finally, the equality $\left(\mathcal{B}_{T}(v), v\right)=0$ implies that $a(x) u_{t}=0$ on $Q_{T}$. If $a(x)>0$ almost everywhere in $\Omega$, then $u_{t}=0$ on $Q_{T}$, then since $u(\cdot, 0)=0$ on $\Omega$, this implies that $u=0$ in $Q_{T}$, then $L u=0$ in $Q_{T}$ then $\sqrt{a(x)} v=0$ in $Q_{T}$ and then finally $v=0$ in $Q_{T}$. The same conclusion holds if $\operatorname{supp} a \neq \Omega$ (for instance $a(x)=1_{\omega}(x)$, $\omega \subset \Omega)$.

\subsection{Internal damping term: a numerical illustration in 1D}

We consider $\Omega=(0,1), 2 a(x)=1_{\omega}(x)$ with $\omega=(0.1,0.3)$. The minimal time for which the observability inequality holds is $T_{o b s}=1.4$. Proceeding as in $[7,21]$, we obtain the following approximation of the spectral abscissa (which is egal to $w(\sqrt{2 a})$, see [12]):

$$
\mu(\mathcal{A})=\sup \{\operatorname{Re}(\lambda) ; \lambda \in \sigma(\mathcal{A})\}=\approx-7.131 \times 10^{-2}
$$

where $\sigma(\mathcal{A})$ denotes the spectrum of $\mathcal{A}$.

Tables 1 collects some values of $k_{T}$ with respect to $T \geq 1.4$. In particular, we check that for $T$ large enough, $k_{T}$ behaves like $1 / T$ for $T$ large. Similarly, Table 2 collects some values of $\widetilde{k_{T}}$ with respect to $T$, for which we observe that $\widetilde{k_{T}}$ behaves likes $T^{2}$ for $T$ large. 


\begin{tabular}{|c|cccccccccc|}
\hline$T$ & 1.4 & 1.405 & 1.41 & 1.42 & 1.45 & 1.5 & 1.75 & 2 & 2.5 & 3 \\
\hline$k_{T}$ & 546.66 & 298.74 & 187.95 & 100.41 & 41.336 & 25.52 & 14.50 & 7.033 & 6.724 & 5.564 \\
\hline
\end{tabular}

\begin{tabular}{|c|ccccccccccc|}
\hline$T$ & 4 & 5 & 6 & 8 & 10 & 12 & 14 & 16 & 20 & 25 & 30 \\
\hline$k_{T}$ & 3.516 & 2.98 & 2.344 & 1.758 & 1.406 & 1.172 & 1.004 & 0.879 & 0.703 & 0.563 & 0.468 \\
\hline
\end{tabular}

Table 1: $k_{T}$ vs. $T \geq 1.4$.

\begin{tabular}{|c|ccccccccc|}
\hline$T$ & 1.4 & 1.45 & 1.5 & 1.75 & 2 & 2.5 & 4 & 8 & 16 \\
\hline$\widehat{k}_{T}$ & 0.112 & 0.128 & 0.137 & 0.187 & 0.243 & 0.377 & 0.966 & 3.540 & 11.369 \\
\hline
\end{tabular}

Table $2: \widetilde{k_{T}}$ vs. $T \geq 1.4$.

Consequently, if we note

$$
C_{T}:=\frac{1}{2 T} \ln \left(1-\frac{1}{k_{T}\left(1+\widetilde{k_{T}}\right)}\right)
$$

we get the values reported in Table 3 . We check, in agreement with the estimate (1.17), that

\begin{tabular}{|c|c|c|c|c|c|c|c|c|c|}
\hline$T$ & \multicolumn{2}{|c|}{1.4} & 1. & & 1 & 5 & & 75 & \\
\hline$C_{T}$ & \multicolumn{2}{|c|}{$-5.88 \times 10^{-4}$} & -7.47 & $<10^{-3}$ & -1.168 & $\times 10^{-2}$ & -1.71 & $\times 10^{-2}$ & $-3.03>$ \\
\hline & $T$ & & .5 & & 4 & & & 1 & \\
\hline & $C_{T}$ & -2.2 & $\times 10^{-2}$ & -1.9 & $\times 10^{-2}$ & -8.36 & $10^{-3}$ & -3.01 & $10^{-3}$ \\
\hline
\end{tabular}

Table 3: $C_{T}$ vs. $T$ for $\Omega=(0,1)$ and $\omega=(0.1,0.3)$.

$$
\mu(\mathcal{A}) \approx-7.131 \times 10^{-2} \leq \inf _{T \geq 1.4} C_{T} \approx-3.03 \times 10^{-2}
$$

and that $\inf _{T \geq 1.4} C_{T}$ provides a satisfactory upper estimate of $\mu(\mathcal{A})$.

\subsection{Internal damping term: numerical illustrations in $2 \mathrm{D}$}

We now consider the two dimensional case: first, we assume that $\Omega$ is the unit square, $\Omega=(0,1)$, and $2 a(x)=101_{\omega}(x)$ with

$$
\omega=\left\{\left(x_{1}, x_{2}\right) \in \Omega, x_{1} \in(0.1,0.3), x_{2} \in(0,1)\right\} \cup\left\{\left(x_{1}, x_{2}\right) \in \Omega, x_{1} \in(0 ., 1 .), x_{2} \in(0.1,0.3)\right\} .
$$

The minimal time for the observability inequality holds is $T_{\text {obs }}=7 \sqrt{2} / 5 \approx 1.97$.

Proceeding as in [21], we obtain the following approximation of the corresponding spectral abcsissa :

$$
\mu(\mathcal{A}) \approx-9.91 \times 10^{-1} .
$$

Similarly, Table 4 reports the values of $C_{T}$ we obtain for $T \leq T_{\text {obs }}$ : Again, in agreement with the estimate (1.17), we observe that $\inf _{T \geq T_{\text {obs }}} C_{T}$ is a reasonable estimation by above of $\mu(\mathcal{A})$, obtained for $T$ around $T=2.3: \inf _{T \geq T_{\text {obs }}} C_{T} \approx-5.2 \times 10^{-1}$.

Finally, we have repeated the computations for the geometry $\Omega$, subset of $(0,1)^{2}$ described on Figure 1 (Left), considered in [21]. The support $\omega$ of the damping function is given on Figure 1 


\begin{tabular}{|c|ccccc|}
\hline$T$ & 2. & 2.1 & 2.2 & 2.25 & 2.3 \\
\hline$C_{T}$ & $-8.2 \times 10^{-2}$ & $-1.8 \times 10^{-1}$ & $-2.8 \times 10^{-1}$ & $-4.1 \times 10^{-1}$ & $-5.2 \times 10^{-1}$ \\
\hline
\end{tabular}

\begin{tabular}{|c|ccccc|}
\hline$T$ & 2.35 & 2.4 & 2.5 & 3 & 4 \\
\hline$C_{T}$ & $-3.5 \times 10^{-1}$ & $-7.6 \times 10^{-2}$ & $-3.9 \times 10^{-3}$ & $-2.1 \times 10^{-3}$ & $-7.32 \times 10^{-4}$ \\
\hline
\end{tabular}

Table 4: $C_{T}$ vs. $T$ for $\Omega=(0,1)^{2}$ and $\omega$ defined by (5.53).
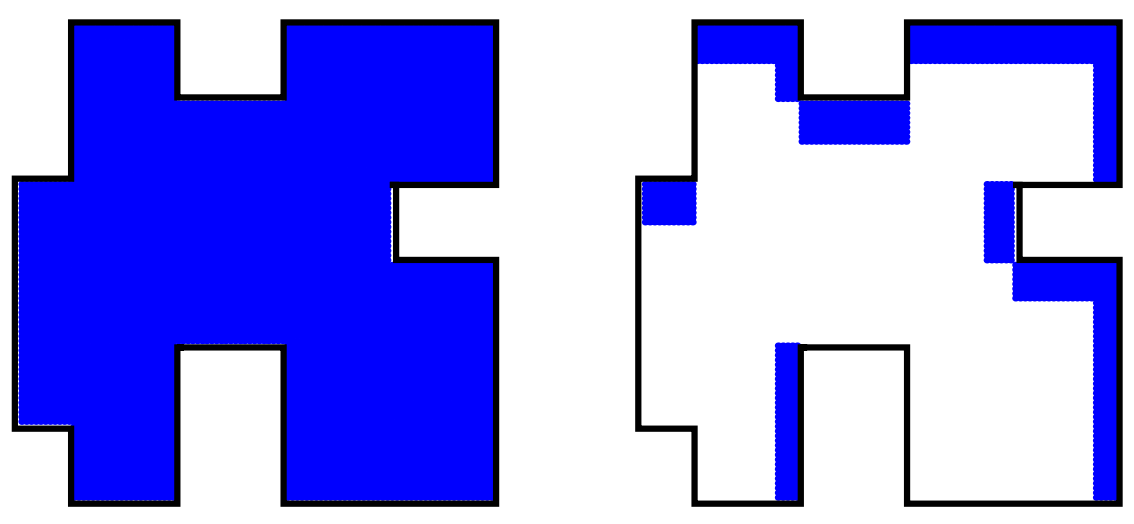

Figure 1: Domain $\Omega \subset(0,1)^{2}$ (left) and support $\omega \subset \Omega$ (right).

(right). For such subdomain $\omega$, the controllability cost $k_{T}$, introduced in (1.14) is finite as soon as $T$ is large enough ( of the order $T_{o p} \approx 2$ here). With again, $a(x)=101_{\omega}(x)$, we compute in $[21] \mu(\mathcal{A}) \approx-1.89 \times 10^{-1}$, while the computation of various $C_{T}$ leads to $\inf _{T} C_{T} \approx-7.49 \times 10^{-2}$ reached approximatively for $T=3.1$.

\section{Appendix: evaluation of the constant in 1D using Fourier Decom- position}

\subsection{Evaluation of $k_{T}$ in 1-D: internal damping case}

For some simple geometry, we may approximate the constant $k_{T}$, which appears in (5.31), by explicit Fourier representation of the homogeneous solution $\varphi$ of (5.32). We assume for simplicity that the damping function is constant such that $2 a(x)=1, x \in \omega \subset(0,1)$.

We first note $\left(a_{p}, b_{p}\right)_{p>0}$ the Fourier coefficients in $l^{2}(\mathbb{N}) \times h^{-1}(\mathbb{N})$ of the initial state $\left(\varphi_{0}, \varphi_{1}\right) \in$ $L^{2}(0,1) \times H^{-1}(0,1)$, such that $\left(\varphi_{0}(x), \varphi_{1}(x)\right)=\sum_{p>0}\left(a_{p}, b_{p}\right) \sin (p \pi x)$. The adjoint state $\varphi$ takes the form

$$
\varphi(x, t)=\sum_{p>0}\left(a_{p} \cos (p \pi t)+\frac{b_{p}}{p \pi} \sin (p \pi t)\right) \sin (p \pi x) .
$$


We get

$$
\begin{aligned}
\iint_{q_{T}} \varphi \bar{\varphi} d x d t & =\sum_{p, q>0} a_{p} \overline{a_{q}} \iint_{q_{T}} \cos (p \pi t) \cos (q \pi t) \sin (p \pi x) \sin (q \pi x) d x d t \\
& +\sum_{p, q>0} a_{p} \overline{b_{q}} \iint_{q_{T}} \cos (p \pi t) \frac{\sin (q \pi t)}{q \pi} \sin (p \pi x) \sin (q \pi x) d x d t \\
& +\sum_{p, q>0} b_{p} \overline{a_{q}} \iint_{q_{T}} \frac{\sin (p \pi t)}{p \pi} \cos (q \pi t) \sin (p \pi x) \sin (q \pi x) d x d t \\
& +\sum_{p, q>0} b_{p} \overline{b_{q}} \iint_{q_{T}} \frac{\sin (p \pi t)}{p \pi} \frac{\sin (q \pi t)}{q \pi} \sin (p \pi x) \sin (q \pi x) d x d t
\end{aligned}
$$

and

$$
\begin{gathered}
\|\varphi(\cdot, 0)\|_{L^{2}(0,1)}^{2}=\sum_{p>0}\left|a_{p}\right|^{2}, \quad\left\|\varphi_{t}(\cdot, 0)\right\|_{H^{-1}(0,1)}^{2}=\sum_{p>0}\left|\frac{b_{p}}{p \pi}\right|^{2} \\
(\varphi(\cdot, 0), \bar{\varphi}(\cdot, 0))_{L^{2}(0,1)}=\sum_{p, q>0} a_{p} \overline{a_{q}}, \quad\left(\varphi_{t}(\cdot, 0), \bar{\varphi}_{t}(\cdot, 0)\right)_{H^{-1}(0,1)}=\sum_{p, q>0} \frac{b_{p} \overline{b_{q}}}{p \pi q \pi}
\end{gathered}
$$

We may therefore introduce a matrix $\mathcal{M}_{q_{T}}$ such that

$$
<\left(\begin{array}{c}
\left\{\overline{a_{q}}\right\}_{q>0} \\
\left\{\overline{b_{q}}\right\}_{q>0}
\end{array}\right), \mathcal{M}_{q_{T}}\left(\begin{array}{c}
\left\{a_{p}\right\}_{p>0} \\
\left\{b_{p}\right\}_{p>0}
\end{array}\right)>=\iint_{q_{T}} \varphi \bar{\varphi} d x d t
$$

and a matrix $\mathcal{B}_{q_{T}}$ such that

$$
\begin{gathered}
<\left(\begin{array}{c}
\left\{\overline{a_{q}}\right\}_{q>0} \\
\left\{\overline{b_{q}}\right\}_{q>0}
\end{array}\right), \mathcal{B}\left(\begin{array}{c}
\left\{a_{p}\right\}_{p>0} \\
\left\{b_{p}\right\}_{p>0}
\end{array}\right)>= \\
(\varphi(\cdot, 0), \bar{\varphi}(\cdot, 0))_{L^{2}(\Omega)}+\left(\varphi_{t}(\cdot, 0), \bar{\varphi}_{t}(\cdot, 0)>_{H^{-1}(\Omega)}\left(=\left\|\varphi_{0}\right\|_{L^{2}(\Omega)}^{2}+\left\|\varphi_{1}\right\|_{H^{-1}(\Omega)}^{2}\right)\right.
\end{gathered}
$$

The inequality (5.31) then rewrite as follows :

$$
<\left(\begin{array}{c}
\left\{\overline{a_{p}}\right\}_{p>0} \\
\left\{\overline{b_{p}}\right\}_{p>0}
\end{array}\right), \mathcal{B}\left(\begin{array}{c}
\left\{a_{p}\right\}_{p>0} \\
\left\{b_{p}\right\}_{p>0}
\end{array}\right)>\leq k_{T}<\left(\begin{array}{c}
\left\{\overline{a_{p}}\right\}_{p>0} \\
\left\{\overline{b_{p}}\right\}_{p>0}
\end{array}\right), \mathcal{M}_{q_{T}}\left(\begin{array}{c}
\left\{a_{p}\right\}_{p>0} \\
\left\{b_{p}\right\}_{p>0}
\end{array}\right)>
$$

for all $\left\{\overline{a_{p}}\right\}_{p>0}$ and $\left\{\overline{b_{p}}\right\}_{p>0}$ and is therefore the solution of the generalized eigenvalue problem

$$
k_{T}=\sup \left\{\lambda: \mathcal{B}\left\{c_{h}\right\}=\lambda \mathcal{M}_{q_{T}}\left\{c_{h}\right\}, \quad \forall\left\{c_{h}\right\}=\left\{a_{h}, b_{h}\right\}^{T} \in \mathbb{R} \backslash\{0\}\right\}
$$

solved using the power iteration method (assuming that the largest eigenvalue is simple). Remark that the matrix $\mathcal{M}_{q_{T}}$ is symmetric, definite positive and full while the matrix $\mathcal{B}$ is diagonal.

\subsection{Evaluation of $\widetilde{k_{T}}$ in 1-D: the internal damping case}

In the $1 \mathrm{D}$ setting, the constant $\widetilde{k_{T}}$ in $(5.43)$ may also be approximated by the way of Fourier expansion. We assume that $v \in L^{2}\left(Q_{T}\right)$ can be written as follows

$$
v(x, t)=\sum_{m, n>0} v_{m, n} f_{m}(t) g_{n}(x), \quad v_{m, n} \in \mathbb{R}
$$

where $\left\{f_{m}\right\}_{m>0}$ and $\left\{g_{n}\right\}_{n>0}$ denote a basis of $L^{2}(0, T)$ and $L^{2}(0,1)$ respectively. 
We then expand the solution of the non homogeneous wave equation as follows: $u(x, t)=$ $\sum_{p>0} b_{p}(t) \sin (p \pi x)$. We get that $b_{p}$ solves the differential equation

$$
\begin{aligned}
b_{q}^{\prime \prime}(t)+(q \pi)^{2} b_{q}(t) & =2 \sum_{m, n} v_{m, n} f_{m}(t) \int_{0}^{1} \sqrt{2 a(x)} g_{n}(x) \sin (q \pi x) d x, \quad q>0 \\
& =\sum_{m, n>0} v_{m, n} c_{n, q} f_{m}(t)
\end{aligned}
$$

with $c_{n, q}=2 \int_{0}^{1} \sqrt{2 a(x)} g_{n}(x) \sin (q \pi x) d x$ leading to

$$
\begin{aligned}
& b_{q}^{\prime}(t)=\sum_{m, n>0} v_{m, n} c_{n, q} \int_{0}^{t} \cos (q \pi(t-s)) f_{m}(s) d s, \\
& u_{t}(x, t)=\sum_{p>0} b_{p}^{\prime}(t) \sin (p \pi x) .
\end{aligned}
$$

Therefore, if we denote by $d_{p, q}(\omega):=\int_{\omega} \sin (p \pi x) \sin (q \pi x) d x$, we have

$$
\int_{\omega} \int_{0}^{T}\left|u_{t}(x, t)\right|^{2} d x d t=\sum_{m, n} \sum_{\bar{m}, \bar{n}} v_{m, n} M_{m, n, \bar{m}, \bar{n}} v_{\bar{m}, \bar{n}}
$$

with

$M_{m, n, \bar{m}, \bar{n}}=\sum_{p, q>0} c_{n, p} c_{\bar{n}, q} d_{p, q}(\omega) \int_{0}^{T}\left(\int_{0}^{t} \cos (p \pi(t-s)) f_{m}(s) d s\right)\left(\int_{0}^{t} \cos (q \pi(t-s)) f_{\bar{m}}(s) d s\right) d t$.

Similarly, we obtain

$$
\|v(x, t)\|_{L^{2}\left(q_{T}\right)}^{2}=\frac{T}{2} \sum_{m, n>0} \sum_{\bar{m}, \bar{n}>0} v_{m, n} \delta_{m, \bar{m}} d_{n, \bar{n}}(\omega) v_{\bar{m}, \bar{n}} .
$$

Eventually, using (6.55) and (6.56), the constant $\widetilde{K}_{T}$ (see (5.43)) can be reformulated as the solution of a generalized eigenvalue problem, as in Section (6.1). In practice, we may use $f_{m}(t)=\sin (m \pi t / T), m>0$ and $g_{n}(x)=\sin (n \pi x), n>0$.

\section{References}

[1] K. Ammari and S. Nicaise, Stabilization of elastic systems by collocated feedback, Lecture Notes in Mathematics, vol. 2124, Springer-Verlag, Berlin, 2015.

[2] K. Ammari, M. Dimassi And M. Zerzeri, The rate at which energy decays in a viscously damped hinged Euler-Bernoulli beam, Journal of Differential Equations, 257 (2014), 35013520 .

[3] K. Ammari, M. Dimassi and M. Zerzeri, Rate of decay of some Petrowsky-like dissipative systems, arXiv:1407.2172.

[4] K. Ammari, A. Henrot, and M. Tucsnak, Asymptotic behaviour of the solutions and optimal location of the actuator for the pointwise stabilization of a string, Asymptot. Anal., 28 (2001), pp. 215-240. 
[5] K. Ammari and M. Tucsnak, Stabilization of second order evolution equations by a class of unbounded feedbacks, ESAIM Control Optim. Calc. Var., 6 (2001), pp. 361-386 (electronic).

[6] K. Ammari, M. Tucsnak, And A. Henrot, Optimal location of the actuator for the pointwise stabilization of a string, C. R. Acad. Sci. Paris Sér. I Math., 330 (2000), pp. 275280 .

[7] M. Asch And G. Lebeau, The spectrum of the damped wave operator for a bounded domain in $\mathbf{R}^{2}$, Experiment. Math., 12 (2003), pp. 227-241.

[8] A. Bensoussan, G. Da Prato, M. C. Delfour, and S. K. Mitter, Representation and control of infinite-dimensional systems. Vol. 1, Systems \& Control: Foundations \& Applications, Birkhäuser Boston Inc., Boston, MA, 1992.

[9] C. Castro and S. J. Cox, Achieving arbitrarily large decay in the damped wave equation, SIAM J. Control Optim., 39 (2001), pp. 1748-1755 (electronic).

[10] F. Chatelin, Spectral approximation of linear operators, Computer Science and Applied Mathematics, Academic Press Inc. [Harcourt Brace Jovanovich Publishers], New York, 1983. With a foreword by P. Henrici, With solutions to exercises by Mario Ahués.

[11] N. CîndeA AND A. MÜNCH, A mixed formulation for the direct approximation of the control of minimal $L^{2}$-norm for linear type wave equations, To appear in Calcolo, http://hal.archives-ouvertes.fr/hal-00853767.

[12] S. Cox And E. Zuazua, The rate at which energy decays in a damped string, Comm. Partial Differential Equations, 19 (1994), pp. 213-243.

[13] G. Doetsch, Introduction to the theory and application of the Laplace transformation, Springer-Verlag, New York, 1974. Translated from the second German edition by Walter Nader.

[14] P. Freitas, Optimizing the rate of decay of solutions of the wave equation using genetic algorithms: a counterexample to the constant damping conjecture, SIAM J. Control Optim., 37 (1999), pp. 376-387 (electronic).

[15] R. Glowinski, J.-L. Lions, And J. He, Exact and approximate controllability for distributed parameter systems, vol. 117 of Encyclopedia of Mathematics and its Applications, Cambridge University Press, Cambridge, 2008. A numerical approach.

[16] A. HARAux, Une remarque sur la stabilisation de certains systèmes du deuxième ordre en temps, Portugal. Math., 46 (1989), pp. 245-258.

[17] A. E. Ingham, Some trigonometrical inequalities with applications to the theory of series, Math. Z., 41 (1936), pp. 367-379.

[18] G. Lebeau, Équation des ondes amorties, in Algebraic and geometric methods in mathematical physics (Kaciveli, 1993), vol. 19 of Math. Phys. Stud., Kluwer Acad. Publ., Dordrecht, 1996, pp. 73-109.

[19] J.-L. Lions, Contrôlabilité exacte, perturbations et stabilisation de systèmes distribués. Tome 1, vol. 8 of Recherches en Mathématiques Appliquées [Research in Applied Mathematics], Masson, Paris, 1988. Contrôlabilité exacte. [Exact controllability], With appendices by E. Zuazua, C. Bardos, G. Lebeau and J. Rauch. 
[20] A. MüNCH, A uniformly controllable and implicit scheme for the 1-D wave equation, M2AN Math. Model. Numer. Anal., 39 (2005), pp. 377-418.

[21] A. MÜNCh AND A. F. PAZOTO, Uniform stabilization of a viscous numerical approximation for a locally damped wave equation, ESAIM Control Optim. Calc. Var., 13 (2007), pp. 265293 (electronic). 